\title{
BMJ Open Promising best practices implemented in long-term care homes during COVID-19 pandemic to address social isolation and loneliness: a scoping review protocol
}

\author{
Idrissa Beogo (D) , ${ }^{1,2}$ Eric Nguemeleu Tchouaket (D), ${ }^{3}$ Drissa Sia (D) , ${ }^{3}$ \\ Nebila Jean-Claude Bationo, ${ }^{4}$ Stephanie Collin, ${ }^{5}$ Diane Tapp, ${ }^{6}$ \\ Said Abasse Kassim (1) , ${ }^{7}$ Jean Ramdé, ${ }^{4}$ Marie-Pierre Gagnon (1) ${ }^{6}$
}

To cite: Beogo I, Tchouaket EN, Sia D, et al. Promising best practices implemented in long-term care homes during COVID-19 pandemic to address social isolation and Ioneliness: a scoping review protocol. BMJ Open 2022;12:e053894. doi:10.1136/ bmjopen-2021-053894

- Prepublication history for this paper is available online. To view these files, please visit the journal online (http://dx.doi. org/10.1136/bmjopen-2021053894).

Received 27 May 2021 Accepted 03 December 2021

Check for updates

(C) Author(s) (or their employer(s)) 2021. Re-use permitted under CC BY-NC. No commercial re-use. See rights and permissions. Published by BMJ.

For numbered affiliations see end of article.

Correspondence to Dr Idrissa Beogo;

ibeogo@uottawa.ca

\section{ABSTRACT}

Introduction Prior to the COVID-19 pandemic, social isolation and loneliness (SIL) affected at least onethird of the older people. The pandemic has prompted governments around the world to implement some extreme measures such as banning public gatherings, imposing social distancing, mobility restrictions and quarantine to control the spread and impact of the novel coronavirus. Though these unprecedented measures may be crucial from a public health perspective, they also have the potential to further exacerbate the problems of SIL among residents in long-term care homes (LTCHs). However, some LTCHs have developed promising best practices (PBPs) to respond to the current situation and prepare for future pandemics. Key aspects of such practices revolve around maintaining and strengthening social connections between residents and their families which helps to reduce SIL. This scoping review looks at existing PBPs that have been implemented to reduce SIL among LTCH residents during the most recent pandemics.

Methods and analysis We will follow Arksey and O'Malley's framework of scoping review, further developed by Levac et al. In addition, we will also apply the Joanna Briggs Institute Reviewers' 'Methodology for Scoping Reviews'. Ten electronic databases and grey literature will be searched for articles published from January 2003 to March 2021 in either English or French. Two reviewers will independently screen titles and abstracts and then full texts for final inclusion. Data will be extracted using a standardised form from 'Evidence for Policy and Practice Information'. The results will be presented in a tabular form and will be summarised and interpreted using a narrative synthesis.

Ethics and dissemination Formal ethical approval is not required as no primary data are collected. Findings will be used to develop a solid knowledge corpus to address the challenges of SIL in LTCHs. Our findings will help to identify cutting edge practices, including technological interventions that could support health services in addressing SIL in the context of LTCHs and our ageing society.
Strengths and limitations of this study

This scoping review will gather promising best practices that have been successfully implemented to combat social isolation and loneliness among residents in long-term care homes to build up a postCOVID-19 surge capacity.

- In addition to the grey literature, this scoping review will explore 10 electronic databases for original academic research papers with search strings that are well calibrated after refined iterative processes to improve the likelihood of retrieving relevant articles.

- We will miss a part of the literature as only English and French published articles will be eligible for inclusion.

- Included articles will not undergo quality assessment, as it is beyond the aim of a scoping review.

\section{INTRODUCTION}

Over the first wave of the COVID-19 pandemic, Canadian long-term care homes (LTCHs) have recorded an unprecedented number of cases and were also the hardest hit in terms of mortality, accounting for about $81 \%$ of the overall COVID-19-related deaths in the country. This proportion almost doubles the Organisation for Economic Co-operation and Development (OECD) countries, ${ }^{1-3}$ average of $42 \%$. Additionally, Canada has also recorded significant provincial-level variations in COVID-19 cases and related deaths. For instance, Shim $^{4}$ reported that by December 2020, the nationwide case fatality rate was $3.36 \%$, while at the provincial level, the adjusted case fatality rate was highest in Québec $(5.13 \%)$-also the province with the highest deaths among older peoplefollowed by Ontario $(3.17 \%)$, British Columbia (1.97\%) and Alberta (1.13\%). 
The very first mortality has caused a shift in our policy priorities amid the pandemic, with more attention being paid to public health interventions and strategies to control the spread and impact of the coronavirus. Like most governments around the world, the current COVID-19 pandemic has prompted the Canadian government to implement some extreme measures including, for instance, banning public gatherings, imposing social distancing, mobility restrictions, isolation and quarantine as part of public health responses. However, the impact of such policy actions on the 'social isolation and loneliness' (SIL) of older people especially those living in LTCHs has been mainly overlooked. It is worth noting that before the COVID-19 pandemic, SIL was reported to be highapproximately 50\%—among Canadians aged 65 and over, particularly those with comorbidities (eg, physical or mental illness, cognitive deficits) or in marginalised groups, and those experiencing life transitions (eg, loss of employment, or a spouse).$^{56}$ The pandemic has further exacerbated this problem by limiting social interactions between residents in LTCHs and their families or loved ones. Families play a fundamental role in caring for older persons, for instance, there is evidence suggesting that one in five Canadian families assist their older members for more than 10 hours/week. ${ }^{7}$ The supportive and interactive culture of LTCHs also encourages families' participation in providing care, including emotional and social support and paying regular visits to their loved ones. ${ }^{8}$ But since such interactions and visits have been enormously disrupted by the pandemic, the SIL of older persons residing in LTCHs is increasingly becoming a concern.

Current statistics indicate that the number of Canadians living in LTCHs has been on the increase. According to the 2016 Canadian census, $6.8 \%$ of Canadians aged 65 years and above were living in a nursing home $(\mathrm{NH})$ or residence for older adults. This proportion jumps to $30.0 \%$ among Canadians aged 85 years and older. ${ }^{910}$ The majority of LTCH residents live with many chronic medical conditions and are more likely to be immune compromised. Thus, the LTCH settings are particularly vulnerable to the spread of infectious diseases such as influenza and other similar respiratory pathogens. ${ }^{11}$ McMichael et al ${ }^{12}$ have previously argued that navigating between multiple NHs was a significant contributing factor in the spread of SARS-CoV-2 infections. This was similar to the conclusion given by Chen et al after analysing the first large-scale geolocational data from 30 million smartphones ${ }^{13}$ where $7 \%$ of smartphones appearing in an $\mathrm{NH}$ also appeared in at least one other facility, even after visits were restricted.

Thus, when the COVID-19 pandemic started, more restrictions have been placed on LTCHs to prevent the further spread of the virus and save lives. But while doing so, more residents are being disconnected from friends and families resulting in perceived isolation and loneliness. As an outcome, SIL arises with a reduction in people's social contacts and networks. Thus, the reduced sociability of the older persons does not start only with age but is also linked first to the reduction of their professional networks, then to the shrinking of their relational networks. ${ }^{14}$ This implies that it is not just age that, in itself, creates isolation but also the social context within which a person aged plays a significant role. Institutionalisation tends to limit individuals' movements leading to a decline in autonomy and contact with established social networks, hence it is considered one of the risk factors for SIL. ${ }^{15}{ }^{16}$ Other risk factors of SIL include, for example, living alone, being unmarried (single, divorced, widowed), not participating in social groups, having fewer friends and strained relationships. ${ }^{17}$

SIL has been identified as a determinant of older people's health ${ }^{18}$ and a risk factor of premature mortality ${ }^{19}$ or for poor physical and mental health, for example, cardiovascular diseases as well as depression and anxiety disorders. ${ }^{20}$ Therefore, the older persons in LTCH need to regularly interact with LTCH staff as well as connect with their families or loved ones. The positive resident-staff interaction involves any non-verbal or verbal exchange ${ }^{21} 22$ that is meaningful for LTCH residents. ${ }^{23}{ }^{24}$ However, interactions like rushing residents during care, juggling concomitantly with several tasks, speaking rapidly and limiting interaction (giving a meal or clearing the table without wording) have the propensity to negatively impact the well-being of residents. ${ }^{25}{ }^{26}$ One recent descriptive cross-sectional study was able to quantify resident-to-staff interactions in 59 assisted livinglesser demanding housing compared with LTCH. ${ }^{27}$ They observed that $39.7 \%$ of interactions occurred in residents' rooms, $31.9 \%$ were care related and $27.4 \%$ were one-on-one sessions. ${ }^{27}$ They also found that as for the type of staff, nursing led accounted for $40.2 \%$ of interactions followed by support staff (eg, dining aide; $24.6 \%$ ).

So far, there has been a dearth of literature on SIL reduction in LTCHs. The two existing reviews ${ }^{28}{ }^{29}$ were both published in the pre-COVID-19 pandemic and have focused on older persons from all settings. In their scoping review, Fakoya $e t a l^{28}$ identified six interventions that have the potential to reduce SIL in older people, namely (1) social facilitation interventions, (2) psychological therapies, (3) health and social care provision, (4) animal interventions, (5) befriending interventions, and (6) leisure/skills development. However, the systematic review by Chipps $e t a l^{29}$ has found inconsistent and weak evidence on using electronic interventions for SIL reduction. The activities offered in NHs to address SIL vary in their composition and the number of persons targeted. Most NHs use a group-based or one-to-one intervention approach $^{30}$ to reduce SIL. Additionally, Theurer $e t a l^{31}$ also identified the use of outings, but other studies also reported strategies such as supporting older people to engage in purposeful activities. ${ }^{32}$

With the start of the COVID-19 pandemic, some of the activities previously used by LTCH to combat SIL (eg, games and outings) have become less effective. ${ }^{33}$ Furthermore, some of the conventional information and communication technology (ICT) platforms (Skype, FaceTime, etc) used by LTCH to connect residents with 
their families ${ }^{34}$ are not that effective either. However, the current COVID-19 pandemic reveals some successful interventions like the use of remote synchronous and asynchronous digital chattering systems, the telephone system or contact behind a glass screen, which allow older people to connect with their families or loved ones.

These are some of the promising best practices (PBPs) that the present scoping review aims to investigate to leverage the reduction of SIL. The review intends to identify the most effective PBPs implemented to address SIL of residents in LTCHs during the present and past pandemics. Given the current COVID-19 pandemic, this review may be considered timely. Importantly, it comes at a time when families, policymakers and care managers need evidence to understand which support strategies and interventions best address the SIL of older people. We are interested in reviewing practices that relate to family support, ICTs, prevention programmes and the role of frontline staff. The review will:

1. Cover the three most recent and important pandemics, namely the SARS, the H1N1 and the current COVID-19 pandemic.

2. Continue the work of Chen and Schulz ${ }^{34}$ published in 2016 on the effect of ICT in reducing SIL of older persons.

3. Identify strategies that LTCHs may implement quickly, during COVID-19 as well as in the post-COVID-19, to strengthen the social connection between older adults residing in LTCHs and their families.

These objectives also align with the urgent needs of managers and stakeholders to support COVID-19 research priorities in Canada and internationally. Thus, this scoping review is part of a larger project funded by the Canadian Institutes for Health Research under the priority programme 'Implementation Science TeamsStrengthening Pandemic Preparedness in Long-Term Care'.

\section{METHODS AND ANALYSIS}

To accomplish the main aim of this study, we will employ the methodological frameworks described by Arksey and $\mathrm{O}^{\prime}$ Malley ${ }^{35}$ as well as Levac et $a l^{36}$ This methodology has six stages: (1) identifying the research question; (2) identifying relevant studies; (3) selecting studies; (4) charting the data; (5) collating, summarising and reporting the results; and (6) consulting knowledge users and developing a knowledge translation (KT) plan involving all stakeholders of the fields to present evidence-informed policy recommendations. The protocol was registered to Research Registry (reviewregistry1157, https://www. researchregistry.com/browse-the-registry\#registryofsy stematicreviewsmeta-analyses/).

\section{Stage 1: identifying the research question}

The research question for this scoping review was developed by a multidisciplinary team in consultation with relevant stakeholders who, at the first wave of the pandemic, participated in assessing variations in COVID-19-related deaths among older persons living in LTCHs in Québec compared with Manitoba and New Brunswick. ${ }^{37} 38$ To identify most relevant studies, our review questions can be summarised as follows:

1. What are the current PBPs implemented in LTCH to alleviate SIL in the older persons during the COVID-19 pandemic and over the previous pandemics, namely SARS and H1N1?

2. How does the use of these best practices reduce the SIL during the COVID-19, SARS and H1N1 among LTCH residents?

Table 1 Inclusion and exclusion criteria: Participants, Interventions, Comparator, Outcome, Time and Setting (PICOTS)

\section{Inclusion criteria}

Participants: Persons $\geq 65$ years (or at least $75 \%$ of sample) living in $\mathrm{LTCH}$, nursing home, care facility settings with no major neurocognitive impairments. A focus will also be put in the minority population including LGBTQ2S+, indigenous population, immigrants, veterans, etc.

Interventions: All kinds of interventions that target social isolation and loneliness to address SIL during the major recent pandemics (COVID-19, H1N1 and SARS). A focus will also be put in e-Interventions. e-Interventions were defined as interventions that are delivered via internet-supported ${ }^{58}$ information and communication technology (ICT) or other electronic technologies, with or without human support. ${ }^{59}$ Outcomes: Qualitative and/or quantitative outcome data focusing on social
isolation and loneliness.

Time: During COVID-19, H1N1 and pandemics.

Settings and study design: Long-term care homes (LTCHs), nursing facilities and assisted living facilities. All study designs will be included (eg, observational studies, randomised controlled trials, reviews and qualitative studies).

Language: English and French language articles published since 2003.

\section{Exclusion criteria}

Participants: Persons with severe neurocognitive disorders, living in community and/or residential settings and with hearing impairment, etc.

Interventions: All types of interventions implemented during seasonal disease outbreak, or implemented in community/ resident settings.

Case management delivered by a trained healthcare professional, information technology (IT)-focused interventions and/or telehealth interventions not focused on SIL.

Outcomes: Physical health outcomes and/or functional capabilities and/or lifestyle changes and no impact on social isolation and/or loneliness.

Pandemic prior to 2003.

Community settings or residential houses.

Any other languages or studies published before 2003. 
3. What lessons, knowledge and recommendations might be learnt and translated into interventions, including the use of digital interventions, to alleviate SIL in LTCH during and after the COVID-19 pandemic?

To follow the recommended steps and operationalise the variables stemming from these research questions, we build up the Participants, Interventions, Comparator, Outcome, Time and Setting (PICOTS) framework $^{3940}$ (table 1). Our scoping review will also be used to gain insight into the use of innovative technologies to support healthcare and health services, while optimally integrating effective value-added technologies for older persons in LTCHs. Note that LTCHs have been identified by Inforoute Santé Canada ${ }^{41}$ to be the least computerised in the Canadian healthcare system.

\section{Stage 2: identifying relevant studies and the grey literature} We will begin by comprehensively mapping academic publications and the grey literature to identify all sources of information relevant to our review questions. To facilitate this stage, we will use the three-step search strategy to retrieve both published and unpublished articles, as recommended by the Joanna Briggs Institute (JBI). ${ }^{42}$ The search will be performed in the English language. A concept plan will be built with the identified keywords and descriptors to perform the query of the databases. The first step has been completed in preparation of this protocol, which involved an initial limited search on Medline followed by an analysis of words taken from the titles, abstracts, keywords and common descriptors (table 2).

A unified search term using Boolean operators was applied for all databases. This approach is consistent with several previous reviews. ${ }^{28} 3043-45$ Ten relevant electronic databases (Medline, EMBASE, CINAHL, Web of Science, PsycInfo, Cochrane, ScienceDirect, DARE, Health Technology and Assessment database, medRxiv) and two grey literature sources (Google Scholar and OpenGrey, Age UK, HelpAge International, Canadian organisations) will be systematically searched for potentially eligible studies published from 2003-corresponding to SARS onsetuntil March 2021, in either English or French language. Backward citation chaining will also be undertaken which will involve hand searching the reference lists of the reviews identified to find other relevant articles. In addition, studies from some highly relevant journals (eg, Ageing \& Society and Journal of Aging \& Social Policy etc) will also be hand searched to ensure literature saturation. Authors of primary studies or reviews may be contacted for further information.

\section{Stage 3: selecting studies and the grey literature}

We specified and refined our inclusion and exclusion criteria based on the PICOTS framework identified for this review (table 2). We will exclude studies that focus on specific subgroups with specific health conditions (eg, physical or sensory disability, specific mental illness, major neurocognitive disorders, etc).

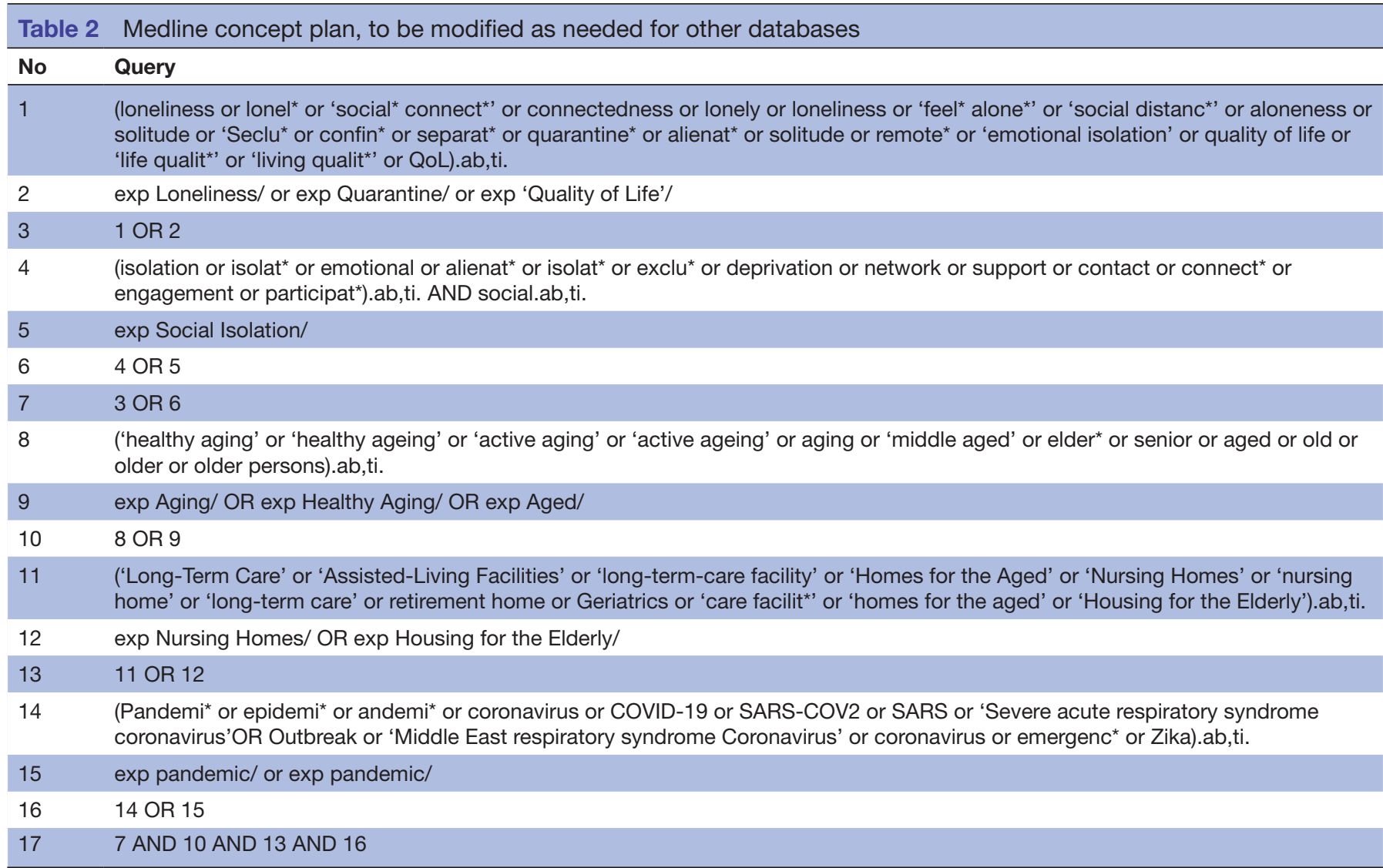




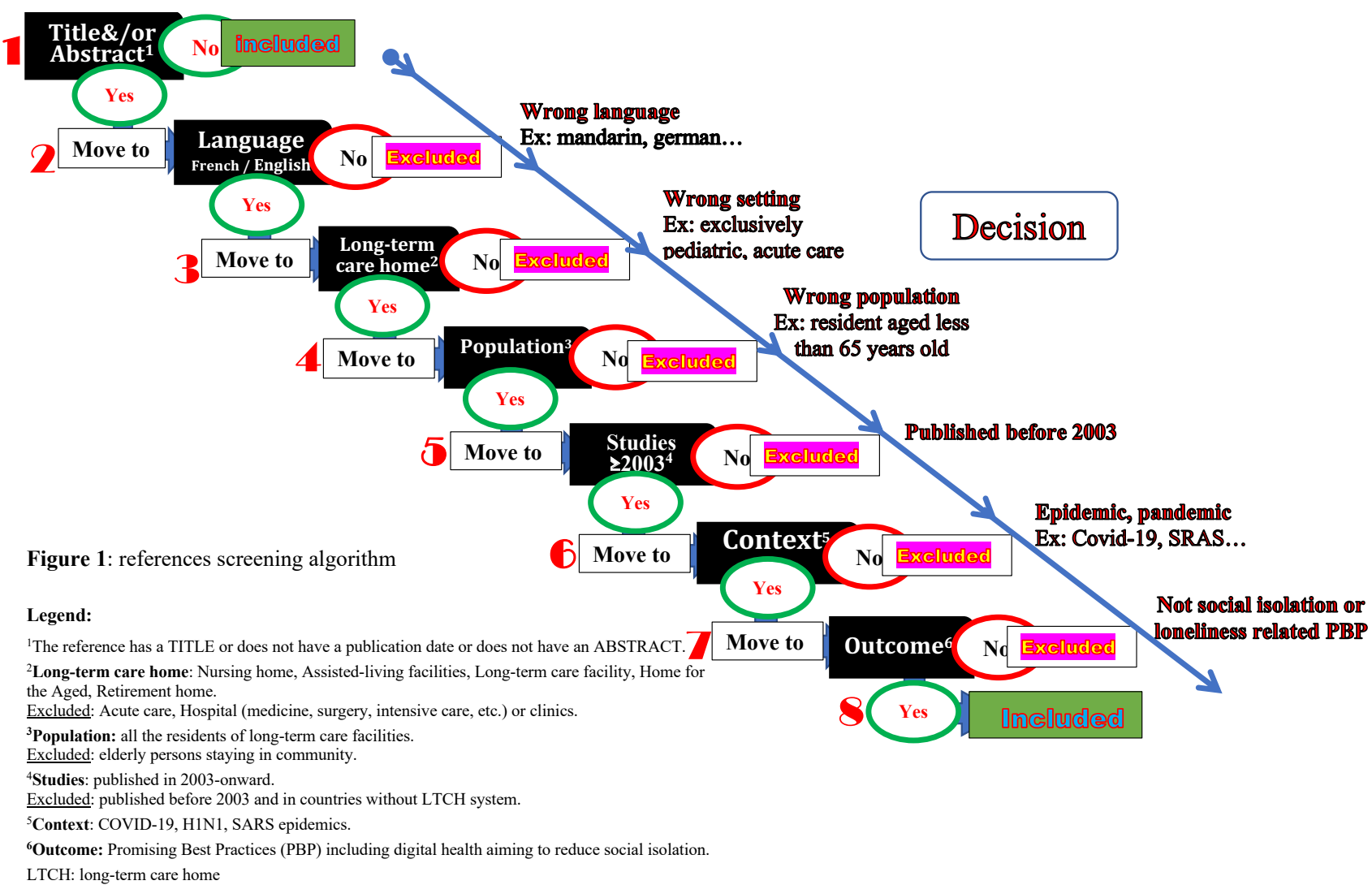

Figure 1 References screening algorithm. LTCH, long-term care home.

Studies are eligible for inclusion if (1) they relate to older adults living in LTCH, (2) the intervention-individual LTCH-level or system-level designed intervention-aimed to alleviate SIL during the aforementioned pandemics, (3) intervention participants were compared against inactive controls and if intervention effects were reported. In this study, the outcome of interest will include structural and functional social support as well as mental and physical health outcomes. Any type of design (eg, observational studies, randomised controlled trials and qualitative studies) will be included.

All retrieved articles will be uploaded into Rayyan Intelligent Systematic Review. ${ }^{46}$ After the removal of duplicates, two reviewers will independently screen the titles and abstracts identified by the literature search for inclusion. The full text of potentially relevant articles will then be obtained and screened to determine the final inclusion. To increase the reliability of screening by the two independent reviewers, a pilot test of the screening based on the eligibility criteria will be performed on a random sample of 230 articles using the algorithm (figure 1) framed and consensually validated by IB, DS and ENT. The $K$ statistic will then be calculated to determine the intrarater agreement for study inclusion. ${ }^{47}$ If necessary, the inclusion and exclusion criteria will be clarified to promote the consistent application of the selection criteria. The involvement of a third reviewer (SC) who is knowledgeable in the research area will be available to resolve discrepancies. Studies excluded during the screening phase will be recorded along with the reason for exclusion by each reviewer.

\section{Stage 4: data charting}

Data extraction will be conducted by NJ-CB (postdoctoral student) including French references (NJ-CB being a native French speaker) under the supervision of the principal investigator (IB). The following information will be extracted using a data extraction form based on a charting table proposed by JBI methodology. ${ }^{42}$ Data extracted will cover the following points: study details (title, authorship, year of publication, location, etc); study design (type of study, duration, outcomes measured, etc); the characteristics of the studied LTCH; participants' demographic (setting, population size and subgroups (eg, minorities, etc)); intervention characteristics (duration, type of intervention categorised according to their mode (eg, social interactions and connections with families and friends, e-Intervention, mixed interventions, or non-e-Intervention, etc)); outcomes (measure of outcomes related to structural social support and functional social support, or any other outcomes related to mental and physical health outcomes, etc); results (raw data and effect size for SIL as the main outcome as well as secondary outcomes); conclusion and interpretation of 
study findings (author and reviewer conclusions, evaluation of the interventions' effects, as well as the barriers and facilitators).

Consistent with the aim of this study, the charting table will maintain a manageable amount of data to examine and map the best practices of interventions designed to alleviate SIL among older people during pandemics or disease outbreaks. Data will be charted by one team member and checked by another. We will not formally appraise methodological quality because a scoping review is meant for identifying gaps in the evidence base and to target areas for future reviews. ${ }^{42}$ Finally, the review will adhere to Preferred Reporting Items for Systematic Reviews and Meta-Analyses for Scoping Reviews(PRISMA-SR) guidelines. ${ }^{48}$

\section{Stage 5: data collating, summary and presentation}

The general characteristics of the included studies will be summarised in tabular form in a manner that reflects the purpose and objective of this scoping review. We expect to find a diverse range of study designs and heterogeneous interventions targeting SIL in older people. Hence, the outcome data regarding the intervention effects will be categorised into validated outcome and non-validated outcome measures. Validated outcome measures are defined as those supported by an academic reference and evidence of their psychometric properties including the Lubben Social Network Scale, University of California, Los Angeles (UCLA) Loneliness Scale.... ${ }^{40}$ Instances of authors using selected items, rather than the full scales of validated measures, led to measures being categorised as partially validated. Non-validated outcome measures are those developed by the authors for the purposes of this study.

A narrative summary will accompany the tabular results and will describe the characteristics of the literature on the interventions targeting SIL among older adults residing in LTCHs during pandemic outbreaks, how the results relate to the review objectives and questions and will identify any gaps in the literature. In case we reach a substantial number of studies selected, we will also adopt an approach similar to vote counting, ${ }^{51-53}$ whereby we will categorise intervention effects as significantly beneficial or not beneficial (table 3 ). In doing so, we can highlight some benefits and limitations associated

Table 3 Vote counting stratified by delivery mode, degree of participation and intervention type

\begin{tabular}{|c|c|c|c|c|c|c|c|c|c|c|c|}
\hline \multirow[b]{2}{*}{ Study } & \multirow[b]{2}{*}{$\begin{array}{l}\text { Delivery } \\
\text { mode }\end{array}$} & \multirow[b]{2}{*}{$\begin{array}{l}\text { Participatory/ } \\
\text { non-participatory }\end{array}$} & \multirow[b]{2}{*}{$\begin{array}{l}\text { Intervention } \\
\text { type }\end{array}$} & \multirow[b]{2}{*}{$\begin{array}{l}\text { Theory } \\
\text { based }\end{array}$} & \multicolumn{4}{|l|}{ Social health } & \multicolumn{2}{|c|}{ Mental health } & \multirow[b]{2}{*}{$\begin{array}{l}\text { Physical } \\
\text { health }\end{array}$} \\
\hline & & & & & Loneliness & $\begin{array}{l}\text { Social } \\
\text { isolation }\end{array}$ & $\begin{array}{l}\text { Structural } \\
\text { social } \\
\text { support }\end{array}$ & $\begin{array}{l}\text { Functional } \\
\text { social } \\
\text { support }\end{array}$ & Depression & $\begin{array}{l}\text { Mental } \\
\text { well-being }\end{array}$ & \\
\hline
\end{tabular}

(20)

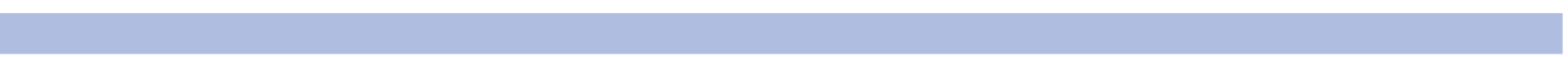

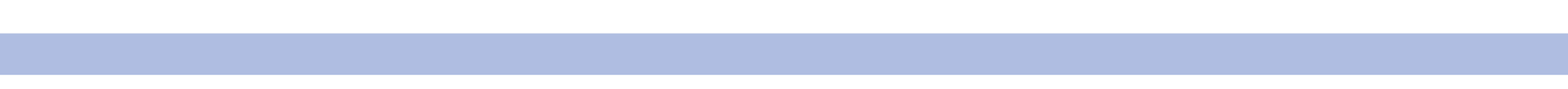

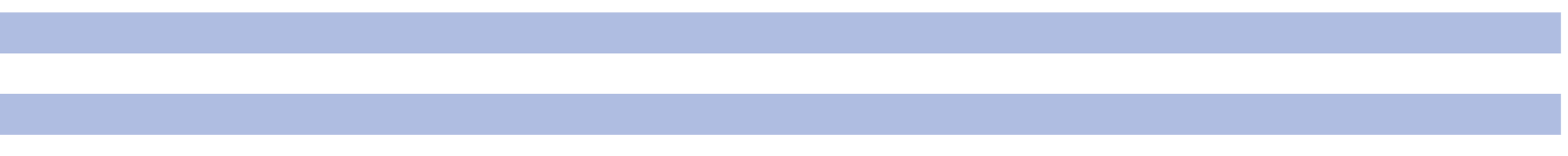

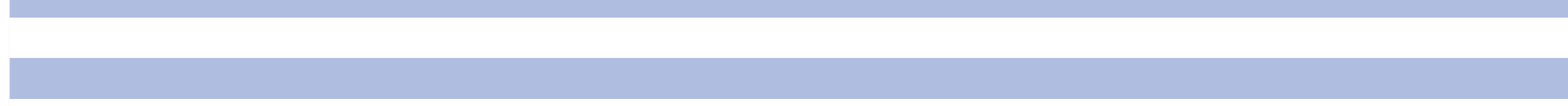

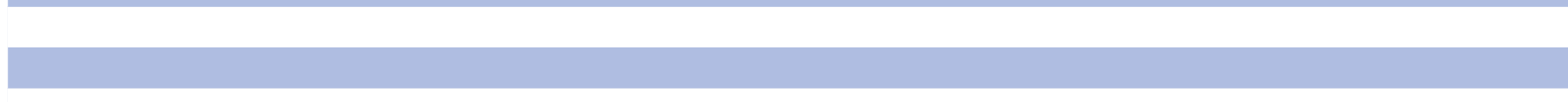


with the use of interventions in minority (eg, linguistic, sexual, cultural) older adults residing in LTCHs during pandemic outbreaks.

\section{Stage 6: consulting knowledge users and developing a KT plan}

This scoping review is part of a larger project on SIL in LTCH. Knowledge stemmed from this scoping review will be employed to guide the implementation of PBP in the LTCH. Our collaborative research built on the co-construction approach will use the Consolidated Framework for Implementation Research (CFIR), ${ }^{54} 55$ working closely with knowledge users (eg, older persons, LTCH managers, families/caregivers, frontline staff and researchers) to collectively create mitigation strategies against SIL in LTCH. By laying out their needs for the PBP, knowledge users have been and will continue to be involved in every step of the review to generate usable and practical findings. This integrated KT model is important for (1) identifying the key interventions arising from this research project; (2) determining the principal target audiences for each of these interventions; (3) seeking out the most credible stakeholders for these interventions and engaging their interest in becoming involved in disseminating and implementing the review findings; and (4) launching a KT strategy grounded in the best available search evidence. We will use a diverse range of approaches to validate and disseminate our preliminary findings to different stakeholders, including an interactive workshop that will bring together the key target audiences for our research. The workshop will explore appropriate strategies and implementation methods and will define success indicators based on the five CFIR domains (intervention characteristics, outer setting, inner setting, characteristics of individuals and process) and by integrating patientpartner aspects, and minorities. In addition, a virtual hackerspace $^{56}$ open to friends of older adults, LTCH users, older adult associations and interested members of the public will be also launched to complement the outcomes of the participatory workshop. This will also be an opportunity to gain insight into the various experiences of digital use interventions by stakeholders (eg, older adult experts, family caregivers, frontline staff) and identify additional practices which are socially acceptable or user-friendly and effective. These strategies will ensure that the research continues to reflect the relevant needs of the end users of this information and to facilitate the appropriate dissemination of outputs.

Meanwhile, some potential challenges related to this scoping review might be anticipated. It might be difficult to categorise the data outcome measures accurately due to the heterogeneity of the interventions (eg, distinguishing between quantitative/qualitative or mixed/ hybrid approaches or those not formally categorised). Second, it might be challenging to interpret the effectiveness of the interventions and categorise them as 'significantly beneficial' or 'not beneficial'. However, we have a strong team with diverse experience in implementation science, nursing health service organisation, digital technologies and research methods, and are planning to hold stakeholder meetings to iteratively receive in-depth feedback from our end users.

\section{DISCUSSION}

Before the COVID-19 pandemic, SIL was a matter of public health programmes, mainly in OECD countries as well as in non-OECD ones (eg, People's Republic of China). The LTCHs were given a little specific consideration in the establishment of measures aimed at countering the effects of a crisis like the COVID-19. ${ }^{20-22}$ Furthermore, it appears that older adults residing in LTCHs, in the context of minority (eg, Lesbian, Gay, Bisexual, Transgender, Queer or Questioning, and Two-Spirit (LGBTQ2S+), indigenous population, immigrants, veterans, etc), ${ }^{17-19}$ are most affected by SIL. As an example, so far, in Ontario (Canada), white Ontarians have experienced the very lowest reported number of the COVID-19 cases, rates of hospitalisation, intensive care unit admission and death, compared with other groups (eg, Latino, Asian, Black...). ${ }^{57}$

The proposed scoping review will be performed to identify the PBP and policies of interventions designed to alleviate SIL in older adults in LTCH. It will draw on the evidence to create an inventory of operationalised practices to reduce the impacts of SIL among older adults in LTCHs during health crises. By mapping and grouping interventions, we intend to determine and propose the type of interventions that are more likely to be effective in LTCHs during future health crises. In doing so, we will discuss and highlight the positive and negative impacts of each selected intervention, as well as the barriers and facilitators that affect their use in LTCHs during a pandemic response. Moreover, we will provide insight into how the use of innovative technologies will be optimised in LTCHs. This review will allow us to produce a strategic action plan that is grounded in knowledge users' feedback and make recommendations on the PBPs and policies designed to alleviate SIL in older adults and provide safe and quality care in LTCHs during health crises.

This scoping review faces some intrinsic limitations regarding the topic and associated context. First, by including only the English and French literature, we might be missing some articles published in other languages. Second, as the current COVID-19 pandemic is still fraught with waves, by targeting COVID-19-related interventions, our review might not be exhaustive since some interventions are still being experimented. Nevertheless, the knowledge generated from this project will guide practices in engaging older adults, families, managers and knowledge users in health innovation research. This research project will ultimately contribute to the development of a solid knowledge based on the impacts and effects of stakeholder engagement in decision-making to mitigate SIL among older adults. Additionally, it will help in promoting the use of innovative technologies 
to support healthcare and health services, while optimally integrating effective value-added technologies for older adults in the health system. The review will help build a strong partnership between knowledge users and researchers, which will be useful for further research. Furthermore, it will contribute to guiding the effectiveness of social interventions for alleviating SIL during the COVID-19 pandemic as well as in postpandemic.

This review will therefore provide policymakers with a better insight into how to tackle SIL by identifying the type of interventions that alleviate or prevent SIL among the older persons in LTCH and under which circumstances. It will generate results that will be highly pertinent to the needs of all knowledge users who are required to implement knowledge or manage changes during a pandemic.

\section{ETHICAL CONSIDERATION}

As this scoping review is part of a 'Social isolation and lonely project', we got ethical approval from the Ethics Committees for Research of the Université de SaintBoniface (ETH 202123 mars), the University of Moncton (dossier 2021-073) and the Research Ethics Board of the Primary Care and Population Health Research Sector of the CIUSSS of the Capitale-Nationale (2021-2303, SPPL).

\section{Author affiliations}

${ }^{1}$ École des sciences infirmières, School of Nursing, Faculty of Health Sciences, University of Ottawa, Ottawa, Ontario, Canada

${ }^{2}$ College of Nursing, Rady Faculty of Health Sciences, University of Manitoba, Winnipeg, Manitoba, Canada

${ }^{3}$ Département Sciences Infirmières, Université du Québec en Outaouais, SaintJerôme, Québec, Canada

${ }^{4}$ Faculté des sciences de l'éducation, Université Laval, Québec, Québec, Canada ${ }^{5}$ École des hautes études publiques, Faculté des arts et des sciences sociales, Université de Moncton, Nouveau Brunswick, Moncton, Canada

${ }^{6}$ Faculté des sciences infirmières, Université Laval, Québec, Québec, Canada

${ }^{7}$ Centre de recherche en gestion des services de santé, Département de management, Faculté des sciences de l'administration, Université Laval, Québec, Québec, Canada

Correction notice This article has been corrected since it was first published. The authors affilation section has been updated.

Acknowledgements This paper is stemmed from the research grant protocol of the PI research programme on ageing and health technology, granted by the Canadian Institutes for Health Research (CIHR) for the programme 'Implementation Science Teams-Strengthening Pandemic Preparedness in Long-Term Care'. We would also like to thank our information specialist Frederic Bergeron for his help in developing the search strategy and selecting databases. We are grateful to the project partners, namely Réseau Compassion Network; Résidence Despins-Villa Aulneau; Jeffery Hale and Saint Brigid's Home (Québec); and Manoir Edith B Pinet and Résidences Lucien Saindon (New Brunswick). Our thanks go to Dr Amadou Darboe for proofreading the manuscript.

Contributors IB conceived the initial idea for the study. Specific contributions were as follows: methodology: IB, M-PG, ENT, DS, SC; validation: JR, DT, SC, M-PG, ENT, DS; project administration: IB, JR, NJ-CB; writing-original draft: IB, SAK, DS, DT; writing-review and editing: IB, ENT, DS, SAK, DT, SC, JR, NJ-CB, M-PG.

Funding This project is funded by the CIHR for the programme 'Implementation Science Teams-Strengthening Pandemic Preparedness in Long-Term Care' (operating grant funds: FRN 174865) (https://webapps.cihr-irsc.gc.ca/decisions/ $\mathrm{p} /$ main.html? lang=en\#fq=B!tag=pinamesDpinamesABeogoldrissaLussierDaniel\& sort=namesortasc\&start=0\&rows $=20$ ).
Disclaimer The CIHR had no role in the process (study design, execution, data analyses or to publish findings).

Competing interests None declared.

Patient and public involvement Patients and/or the public were not involved in the design, or conduct, or reporting, or dissemination plans of this research.

Patient consent for publication Not required.

Provenance and peer review Not commissioned; externally peer reviewed.

Open access This is an open access article distributed in accordance with the Creative Commons Attribution Non Commercial (CC BY-NC 4.0) license, which permits others to distribute, remix, adapt, build upon this work non-commercially, and license their derivative works on different terms, provided the original work is properly cited, appropriate credit is given, any changes made indicated, and the use is non-commercial. See: http://creativecommons.org/licenses/by-nc/4.0/.

\section{ORCID iDs}

Idrissa Beogo http://orcid.org/0000-0003-1467-2169

Eric Nguemeleu Tchouaket http://orcid.org/0000-0002-4309-0478

Drissa Sia http://orcid.org/0000-0003-0044-8465

Said Abasse Kassim http://orcid.org/0000-0003-2136-0825

Marie-Pierre Gagnon http://orcid.org/0000-0002-0782-5457

\section{REFERENCES}

1 Canadian Institute for Health Information. Pandemic experience in the long-term care sector: how does Canada compare with other countries? 2020. Available: https://www.cihi.ca/sites/default/files/ document/covid-19-rapid-response-long-term-care-snapshot-en.pdf

2 Rothan HA, Byrareddy SN. The epidemiology and pathogenesis of coronavirus disease (COVID-19) outbreak. J Autoimmun 2020;109:102433.

3 Grant K. $81 \%$ of COVID-19 deaths in Canada were in long-term care - nearly double OECD average. Globe and Mail 2020.

4 Shim E. Regional variability in COVID-19 case fatality rate in Canada February-December 2020. Int J Environ Res Public Health 2021;18 doi:10.3390/ijerph18041839

5 National Seniors Council. Report on the social isolation of seniors 2013-2014, 2014. Available: file:///Users/idrissabeogo/Downloads/ Report on the Social Isolation of Seniors.pdf

6 National Seniors Council. Who's at risk and what can be done about it? A review of the literature on the social isolation of different groups of seniors, 2017. Available: file://Users/idrissabeogo/Downloads/ nsc1.eng\%20(1).pdf

7 Turcotte M, Sawaya C. Senior care: differences by type of housing catalogue No. 75-006-X statistics Canada. Ottawa, 2015.

8 Bangerter LR, Van Haitsma K, Heid AR, et al. "Make me feel at ease and at home": differential care preferences of nursing home residents. Gerontologist 2016;56:702-13.

9 Statistics Canada. Data tables, 2016 census: age (in single years) and average age and sex for the population - Canada, provinces and territories, census metropolitan areas, and census Agglomerations catalogue 98-400-X2016001) released may 3, 20172017.

10 Statistics Canada [SC]. Data tables, 2016 census: dwelling type, age and sex for the population in occupied dwellings - Canada, provinces and territories, census metropolitan areas, and census agglomerations catalogue 98-400-X2016021 2017.

11 Lansbury LE, Brown CS, Nguyen-Van-Tam JS. Influenza in long-term care facilities. Influenza Other Respir Viruses 2017;11:356-66.

12 McMichael TM, Currie DW, Clark S, et al. Epidemiology of Covid-19 in a long-term care facility in King County, Washington. N Engl J Med 2020;382:2005-11.

13 Chen MK, Chevalier JA, Long EF. Nursing home staff networks and COVID-19. Proc Natl Acad Sci U S A 2021;118:1. doi:10.1073/ pnas.2015455118

14 Bultez J-P. Isolement et exclusion dans Le vieillissement. Economie \& Humanisme 2005;374.

15 Zhou Z, Mao F, Zhang W, et al. The association between loneliness and cognitive impairment among older men and women in China: a nationwide longitudinal study. Int J Environ Res Public Health 2019;16 doi:10.3390/ijerph16162877

16 Prieto-Flores M-E, Forjaz MJ, Fernandez-Mayoralas G, et al. Factors associated with loneliness of noninstitutionalized and institutionalized older adults. J Aging Health 2011;23:177-94.

17 Holt-Lunstad J, Smith TB, Layton JB. Social relationships and mortality risk: a meta-analytic review. PLoS Med 2010;7:e1000316. 
18 Linehan T. 2030 vision: the best and worst futures for older people in the UK, 2014. Available: http://www.ilcuk.org.uk/index.php/ publications/publication_details/2030_vision_the_best_and_worst_ futures for older people in the uk

19 Rico-Uribe LA, Caballero FF, Martín-María N, et al. Association of loneliness with all-cause mortality: a meta-analysis. PLoS One 2018;13:e0190033.

20 Evans IEM, Martyr A, Collins R, et al. Social isolation and cognitive function in later life: a systematic review and meta-analysis. $J$ Alzheimers Dis 2019;70:S119-44.

21 Dean R, Proudfoot R, Lindesay J. The quality of interactions schedule (QUIS): development, reliability and use in the evaluation of two domus units. Int J Geriatr Psychiatry 1993;8:819-26.

22 Machiels M, Metzelthin SF, Hamers JPH, et al. Interventions to improve communication between people with dementia and nursing staff during daily nursing care: a systematic review. Int J Nurs Stud 2017;66:37-46.

23 Hartmann CW, Mills WL, Pimentel CB, et al. Impact of intervention to improve nursing home Resident-Staff interactions and engagement. Gerontologist 2018;58:e291-301.

24 Paudel A, Resnick B, Galik E. The quality of interactions between staff and residents with cognitive impairment in nursing homes. Am J Alzheimers Dis Other Demen 2020;35:153331751986325.

25 Belzil G, Vézina J. Impact of caregivers' behaviors on resistiveness to care and collaboration in persons with dementia in the context of hygienic care: an interactional perspective. Int Psychogeriatr 2015;27:1861-73.

26 Colón-Emeric C, Toles M, Cary MP, et al. Sustaining complex interventions in long-term care: a qualitative study of direct care staff and managers. Implement Sci 2016;11:94.

27 Paudel A, Galik E, Resnick B, et al. A description of Staff-resident interactions in assisted living. Clin Nurs Res 2021;30:690-8.

28 Fakoya OA, McCorry NK, Donnelly M. Loneliness and social isolation interventions for older adults: a scoping review of reviews. BMC Public Health 2020;20:129.

29 Chipps J, Jarvis MA, Ramlall S. The effectiveness of e-Interventions on reducing social isolation in older persons: a systematic review of systematic reviews. J Telemed Telecare 2017;23:817-27.

30 Dickens AP, Richards SH, Greaves CJ, et al. Interventions targeting social isolation in older people: a systematic review. BMC Public Health 2011;11:647.

31 Theurer K, Mortenson WB, Stone R, et al. The need for a social revolution in residential care. J Aging Stud 2015;35:201-10.

32 O'Rourke HM, Collins L, Sidani S. Interventions to address social connectedness and loneliness for older adults: a scoping review. BMC Geriatr 2018;18:214.

33 Theurer K, Mortenson WB, Stone R, et al. The need for a social revolution in residential care. J Aging Stud 2015;35:201-10.

34 Chen Y-RR, Schulz PJ. The effect of information communication technology interventions on reducing social isolation in the elderly: a systematic review. J Med Internet Res 2016;18:e18.

35 Arksey H, O'Malley L. Scoping studies: towards a methodological framework. Int J Soc Res Methodol 2005;8:19-32.

36 Levac D, Colquhoun H, O'Brien KK. Scoping studies: advancing the methodology. Implement Sci 2010;5:69.

37 Nouvelles T. $82 \%$ des décès liés la COVID-19 dans les résidences pour aînés, 2020

38 Béland D, Marier P. COVID-19 and long-term care policy for older people in Canada. J Aging Soc Policy 2020;32:358-64.

39 Schardt C, Adams MB, Owens T, et al. Utilization of the PICO framework to improve searching PubMed for clinical questions. BMC Med Inform Decis Mak 2007;7:16.
40 Samson D, Schoelles KM. Developing the topic and structuring systematic reviews of medical tests: utility of PICOTS, analytic frameworks, decision trees, and other frameworks methods guide for medical test reviews. Rockville, MD, 2012.

41 Inforoute Santé Canada. Sondage national des infirmières et infirmiers du Canada 2020-Utilisation des technologies de santé numériques au travail, 2020. Available: https://infoway-inforoute. $\mathrm{ca} / \mathrm{en} / \mathrm{component/edocman/resources/reports/benefits-evaluation/}$ 3812-2020-national-survey-of-canadian-nurses-use-of-digitalhealth-technology-in-practice

42 Peters MDJ, Marnie C, Tricco AC, et al. Updated methodological guidance for the conduct of scoping reviews. JBI Evid Synth 2020;18:2119-26.

43 Poscia A, Stojanovic J, La Milia DI, et al. Interventions targeting loneliness and social isolation among the older people: an update systematic review. Exp Gerontol 2018;102:133-44.

44 Landeiro F, Barrows P, Nuttall Musson E, et al. Reducing social isolation and loneliness in older people: a systematic review protocol. BMJ Open 2017;7:e013778.

45 Sander R. Preventing social isolation and loneliness among older people: a systematic review of health promotion interventions. Nurs Older People 2005;17:40.

46 Ouzzani M, Hammady H, Fedorowicz Z, et al. Rayyan-a web and mobile APP for systematic reviews. Syst Rev 2016;5.

47 Landis JR, Koch GG. The measurement of observer agreement for categorical data. Biometrics 1977;33:159-74.

48 Shamseer L, Moher D, Clarke M, et al. Preferred reporting items for systematic review and meta-analysis protocols (PRISMA-P) 2015: elaboration and explanation. BMJ 2015;349:g7647

49 Lubben JE. Assessing social networks among elderly populations. Fam Community Health 1988;11:42-52.

50 Russell D, Peplau LA, Cutrona CE. The revised UCLA loneliness scale: concurrent and discriminant validity evidence. J Pers Soc Psychol 1980;39:472-80.

51 Voils Cl, Sandelowski M, Barroso J, et al. Making sense of qualitative and quantitative findings in mixed research synthesis studies. Field methods 2008;20:3-25.

52 Sandelowski M, Voils Cl, Leeman J, et al. Mapping the mixed methods-mixed research synthesis terrain. J Mix Methods Res 2012;6:317-31.

53 Hedges LV, Olkin I. Vote-counting methods in research synthesis. Psychol Bull 1980;88:359-69.

54 Damschroder L, Hall C, Gillon L, et al. The consolidated framework for implementation research (CFIR): progress to date, tools and resources, and plans for the future. Implement Sci 2015;10:A12.

55 Breimaier HE, Heckemann B, Halfens RJG, et al. The consolidated framework for implementation research (CFIR): a useful theoretical framework for guiding and evaluating a guideline implementation process in a hospital-based nursing practice. BMC Nurs 2015;14:1-9.

56 Dunbar-Hester C. Hacking diversity - the politics of inclusion in open technology cultures. Princeton University Press, 2020.

57 McKenzie K, Dube S, Petersen S. Tracking COVID-19 through racebased data, 2021. Available: https://www.wellesleyinstitute.com/wpcontent/uploads/2021/08/Tracking-COVID-19-Through-Race-BasedData eng.pdf

58 Barak A, Klein B, Proudfoot JG. Defining internet-supported therapeutic interventions. Ann Behav Med 2009;38:4-17.

59 Barak A, Hen L, Boniel-Nissim M, et al. A comprehensive review and a meta-analysis of the effectiveness of internetbased psychotherapeutic interventions. J Technol Hum Serv 2008;26:109-60. 